\title{
A Concise Tool for Measuring Care Coordination from the Provider's Perspective in the Hospital Setting
}

\author{
Christine M. Weston, $\mathrm{PhD}^{1}$ and Sehyo Yune, MD², Eric B. Bass, MD, MPH ${ }^{3,4}$, Scott A. Berkowitz, MD, MBA \\ Daniel J. Brotman, $\mathrm{MD}^{6}$, Amy Deutschendorf, MS, RN, ACNS-BC ${ }^{7}$, Eric E. Howell, MD , Melissa B. Richardson, MBA7, \\ Carol Sylvester, RN, $\mathrm{MS}^{9}$, Albert W. Wu, MD, MPH ${ }^{1 *}$
}

\begin{abstract}
${ }^{1}$ Department of Health Policy and Management, Bloomberg School of Public Health, Johns Hopkins University, Baltimore, Maryland; ${ }^{2}$ Bloomberg School of Public Health, Johns Hopkins University, Baltimore, Maryland; ${ }^{3}$ Evidence-Based Practice Center, Bloomberg School of Public Health, Johns Hopkins University, Baltimore, Maryland; ' ${ }^{4}$ epartment of Internal Medicine, Johns Hopkins School of Medicine, Baltimore, Maryland; ${ }^{5}$ Department of Cardiology, Johns Hopkins School of Medicine, Baltimore, Maryland; ${ }^{6}$ Department of Medicine, Johns Hopkins Hospital, Baltimore, Maryland; ${ }^{7}$ Care Coordination and Clinical Resource Management, Johns Hopkins Hospital, Baltimore, Maryland; ${ }^{8}$ Department of Medicine, Johns Hopkins Bayview Medical Center, Baltimore, Maryland; ${ }^{9}$ Care Management Services, Johns Hopkins Bayview Medical Center, Baltimore, Maryland.
\end{abstract}

BACKGROUND: To support hospital efforts to improve coordination of care, a tool is needed to evaluate care coordination from the perspective of inpatient healthcare professionals.

OBJECTIVES: To develop a concise tool for assessing care coordination in hospital units from the perspective of healthcare professionals, and to assess the performance of the tool in measuring dimensions of care coordination in 2 hospitals after implementation of a care coordination initiative.

METHODS: We developed a survey consisting of 12 specific items and 1 global item to measure provider perceptions of care coordination across a variety of domains, including teamwork and communication, handoffs, transitions, and patient engagement. The questionnaire was distributed online between October 2015 and January 2016 to nurses, physicians, social workers, case managers, and other professionals in 2 tertiary care hospitals.

RESULTS: A total of 841 inpatient care professionals completed the survey (response rate $=56.6 \%$ ). Among respondents, 590 $(75 \%)$ were nurses and $37(4.7 \%)$ were physicians. Exploratory factor analysis revealed 4 subscales: (1) Teamwork, (2) Patient Engagement, (3) Handoffs, and (4) Transitions (Cronbach's alpha 0.84-0.90). Scores were fairly consistent for 3 subscales but were lower for patient engagement. There were minor differences in scores by profession, department, and hospital.

CONCLUSION: The new tool measures 4 important aspects of inpatient care coordination with evidence for internal consistency and construct validity, indicating that the tool can be used in monitoring, evaluating, and planning care coordination activities in hospital settings. Journal of Hospital Medicine 2017;12:811-817. Published online first August 23, 2017. (C) 2017 Society of Hospital Medicine
Care Coordination has been defined as "...the deliberate organization of patient care activities between two or more participants (including the patient) involved in a patient's care to facilitate the appropriate delivery of healthcare services." The Institute of Medicine identified care coordination as a key strategy to improve the American healthcare system, ${ }^{2}$ and evidence has been building that well-coordinated care improves patient outcomes and reduces healthcare costs associated with chronic conditions..$^{3-5}$ In 2012, Johns Hopkins Medicine was awarded a Healthcare Innovation Award by the Centers for Medicare \& Medicaid Services to improve coordination of care across the continuum of care for adult patients admitted to Johns Hopkins Hospital (JHH) and

\footnotetext{
*Address for correspondence and reprint requests: Albert W. Wu, MD, MPH 624 N Broadway, Baltimore, MD 21205; Telephone: 410-955-6567; Fax: 410955-0470; E-mail: awu@jhu.edu

Additional Supporting Information may be found in the online version of this article.

Received: February 7, 2017; Revised: April 17, 2017;

Accepted: April 24, 2017
}

2017 Society of Hospital Medicine DOI: 10.12788/jhm.2795
Johns Hopkins Bayview Medical Center (JHBMC), and for high-risk low-income Medicare and Medicaid beneficiaries receiving ambulatory care in targeted zip codes. The purpose of this project, known as the Johns Hopkins Community Health Partnership (J-CHiP), was to improve health and healthcare and to reduce healthcare costs. The acute care component of the program consisted of a bundle of interventions focused on improving coordination of care for all patients, including a "bridge to home" discharge process, as they transitioned back to the community from inpatient admission. The bundle included the following: early screening for discharge planning to predict needed postdischarge services; discussion in daily multidisciplinary rounds about goals and priorities of the hospitalization and potential postdischarge needs; patient and family self-care management; education enhanced medication management, including the option of "medications in hand" at the time of discharge; postdischarge telephone follow-up by nurses; and, for patients identified as high-risk, a "transition guide" (a nurse who works with the patient via home visits and by phone to optimize compliance with care for 30 days postdischarge). ${ }^{6}$ While the primary endpoints of the J-CHiP program were to improve clinical outcomes and reduce healthcare costs, 
we were also interested in the impact of the program on care coordination processes in the acute care setting. This created the need for an instrument to measure healthcare professionals' views of care coordination in their immediate work environments.

We began our search for existing measures by reviewing the Coordination Measures Atlas published in 2014. ${ }^{7} \mathrm{Al}$ though this report evaluates over 80 different measures of care coordination, most of them focus on the perspective of the patient and/or family members, on specific conditions, and on primary care or outpatient settings. ${ }^{7,8}$ We were unable to identify an existing measure from the provider perspective, designed for the inpatient setting, that was both brief but comprehensive enough to cover a range of care coordination domains. ${ }^{8}$

Consequently, our first aim was to develop a brief, comprehensive tool to measure care coordination from the perspective of hospital inpatient staff that could be used to compare different units or types of providers, or to conduct longitudinal assessment. The second aim was to conduct a preliminary evaluation of the tool in our healthcare setting, including to assess its psychometric properties, to describe provider perceptions of care coordination after the implementation of $\mathrm{J}$-CHiP, and to explore potential differences among departments, types of professionals, and between the 2 hospitals.

\section{METHODS}

\section{Development of the Care Coordination Questionnaire}

The survey was developed in collaboration with leaders of the J-CHiP Acute Care Team. We met at the outset and on multiple subsequent occasions to align survey domains with the main components of the J-CHiP acute care intervention and to assure that the survey would be relevant and understandable to a variety of multidisciplinary professionals, including physicians, nurses, social workers, physical therapists, and other health professionals. Care was taken to avoid redundancy with existing evaluation efforts and to minimize respondent burden. This process helped to ensure the content validity of the items, the usefulness of the results, and the future usability of the tool.

We modeled the Care Coordination Questionnaire (CCQ) after the Safety Attitudes Questionnaire (SAQ), ${ }^{9}$ a widely used survey that is deployed approximately annually at JHH and JHBMC. While the SAQ focuses on healthcare provider attitudes about issues relevant to patient safety (often referred to as safety climate or safety culture), this new tool was designed to focus on healthcare professionals' attitudes about care coordination. Similar to the way that the SAQ "elicits a snapshot of the safety climate through surveys of frontline worker perceptions," we sought to elicit a picture of our care coordination climate through a survey of frontline hospital staff.

The CCQ was built upon the domains and approaches to care coordination described in the Agency for Healthcare Research and Quality Care Coordination Atlas. ${ }^{3}$ This report identifies 9 mechanisms for achieving care coordina- tion, including the following: Establish Accountability or Negotiate Responsibility; Communicate; Facilitate Transitions; Assess Needs and Goals; Create a Proactive Plan of Care; Monitor, Follow Up, and Respond to Change; Support Self-Management Goals; Link to Community Resources; and Align Resources with Patient and Population Needs; as well as 5 broad approaches commonly used to improve the delivery of healthcare, including Teamwork Focused on Coordination, Healthcare Home, Care Management, Medication Management, and Health IT-Enabled Coordination. ${ }^{7}$ We generated at least 1 item to represent 8 of the 9 domains, as well as the broad approach described as Teamwork Focused on Coordination. After developing an initial set of items, we sought input from 3 senior leaders of the J-CHiP Acute Care Team to determine if the items covered the care coordination domains of interest, and to provide feedback on content validity. To test the interpretability of survey items and consistency across professional groups, we sent an initial version of the survey questions to at least 1 person from each of the following professional groups: hospitalist, social worker, case manager, clinical pharmacist, and nurse. We asked them to review all of our survey questions and to provide us with feedback on all aspects of the questions, such as whether they believed the questions were relevant and understandable to the members of their professional discipline, the appropriateness of the wording of the questions, and other comments. Modifications were made to the content and wording of the questions based on the feedback received. The final draft of the questionnaire was reviewed by the leadership team of the J-CHiP Acute Care Team to ensure its usefulness in providing actionable information.

The resulting 12-item questionnaire used a 5-point Likert response scale ranging from $1=$ "disagree strongly" to $5=$ "agree strongly," and an additional option of "not applicable (N/A)." To help assess construct validity, a global question was added at the end of the questionnaire asking, "Overall, how would you rate the care coordination at the hospital of your primary work setting?" The response was measured on a 10-point Likert-type scale ranging from 1 = "totally uncoordinated care" to $10=$ "perfectly coordinated care" (see Appendix). In addition, the questionnaire requested information about the respondents' gender, position, and their primary unit, department, and hospital affiliation.

\section{Data Collection Procedures}

An invitation to complete an anonymous questionnaire was sent to the following inpatient care professionals: all nursing staff working on care coordination units in the departments of medicine, surgery, and neurology/neurosurgery, as well as physicians, pharmacists, acute care therapists (eg, occupational and physical therapists), and other frontline staff. All healthcare staff fitting these criteria was sent an e-mail with a request to fill out the survey online using Qualtrics $^{\mathrm{TM}}$ (Qualtrics Labs Inc., Provo, UT), as well as multiple follow-up reminders. The participants worked either at the 
JHH (a 1194-bed tertiary academic medical center in Baltimore, MD) or the JHBMC (a 440-bed academic community hospital located nearby). Data were collected from October 2015 through January 2016.

\section{Analysis}

Means and standard deviations were calculated by treating the responses as continuous variables. We tried 3 different methods to handle missing data: (1) without imputation, (2) imputing the mean value of each item, and (3) substituting a neutral score. Because all 3 methods produced very similar results, we treated the N/A responses as missing values without imputation for simplicity of analysis. We used STATA 13.1 (Stata Corporation, College Station, Texas) to analyze the data.

To identify subscales, we performed exploratory factor analysis on responses to the 12 specific items. Promax rotation was selected based on the simple structure. Subscale scores for each respondent were generated by computing the mean of responses to the items in the subscale. Internal consistency reliability of the subscales was estimated using Cronbach's alpha. We calculated Pearson correlation coefficients for the items in each subscale, and examined Cronbach's alpha deleting each item in turn. For each of the subscales identified and the global scale, we calculated the mean, standard deviation, median and interquartile range. Although distributions of scores tended to be non-normal, this was done to increase interpretability. We also calculated percent scoring at the ceiling (highest possible score).

We analyzed the data with 3 research questions in mind: Was there a difference in perceptions of care coordination between (1) staff affiliated with the 2 different hospitals, (2) staff affiliated with different clinical departments, or (3) staff with different professional roles? For comparisons based on hospital and department, and type of professional, nonparametric tests (Wilcoxon rank-sum and Kruskal-Wallis test) were used with a level of statistical significance set at 0.05 . The comparison between hospitals and departments was made only among nurses to minimize the confounding effect of different distribution of professionals. We tested the distribution of "years in specialty" between hospitals and departments for this comparison using Pearson's $\chi^{2}$ test. The difference was not statistically significant $(P=0.167$ for hospitals, and $P=0.518$ for departments), so we assumed that the potential confounding effect of this variable was negligible in this analysis. The comparison of scores within each professional group used the Friedman test. Pearson's $\chi^{2}$ test was used to compare the baseline characteristics between 2 hospitals.

\section{RESULTS}

Among the 1486 acute care professionals asked to participate in the survey, 841 completed the questionnaire (response rate $56.6 \%$ ). Table 1 shows the characteristics of the participants from each hospital. Table 2 summarizes the item response rates, proportion scoring at the ceiling, and weighting from the factor analysis. All items had completion rates of $99.2 \%$ or higher, with N/A responses ranging from $0 \%$ (item 2) to $3.1 \%$ (item 7 ). The percent scoring at the ceiling was $1.7 \%$ for the global item and ranged from $18.3 \%$ up to $63.3 \%$ for other individual items.

Factor analysis yielded 3 factors comprising 6, 3, and 2 items, respectively. Item 7 did not load on any of the 3 factors, but was retained as a subscale because it represented a distinct domain related to care coordination. To describe these domains, factor 1 was named the "Teamwork" subscale; factor 2, "Patient Engagement"; factor 3, "Transitions"; and item 7, "Handoffs." Subscale scores were calculated as the mean of item response scale scores. An overall scale score was also calculated as the mean of all 12 items. Average inter-item correlations ranged from 0.417 to 0.778 , and Cronbach alpha was greater than 0.84 for the 3 multi-item subscales (Table 2). The pairwise correlation coefficients between the four subscales ranged from 0.368 (Teamwork and Handoffs) to 0.581 (Teamwork and Transitions). The correlation coefficient with the global item was 0.714 for Teamwork, 0.329 for Handoffs, 0.561 for Patient Engagement, 0.617 for Transitions, and 0.743 for overall scale. The percent scoring at the ceiling was $10.4 \%$ to $34.0 \%$ for subscales.

We used the new subscales to explore the perception of inpatient care coordination among healthcare professionals that were involved in the J-CHiP initiative $(\mathrm{n}=646)$. Table 3 shows scores for respondents in different disciplines, comparing nurses, physicians and others. For all disciplines, participants reported lower levels of coordination on $\mathrm{Pa}$ tient Engagement compared to other subscales $(P<0.001$ for nurses and others, $P=0.0011$ for physicians). The mean global rating for care coordination was 6.79 on the 1 to 10 scale. There were no significant differences by profession on the subscales and global rating.

Comparison by hospital and primary department was carried out for nurses who comprised the largest proportion of respondents (Figure). The difference between hospitals on the transitions subscale was of borderline significance ( 4.24 vs $4.05 ; P=0.051)$, and was significant in comparing departments to one another $(4.10,4.35$, and 4.12 , respectively for medicine, surgery, and others; $P=0.002$ ).

We also examined differences in perceptions of care coordination among nursing units to illustrate the tool's ability to detect variation in Patient Engagement subscale scores for JHH nurses (see Appendix).

\section{DISCUSSION}

This study resulted in one of the first measurement tools to succinctly measure multiple aspects of care coordination in the hospital from the perspective of healthcare professionals. Given the hectic work environment of healthcare professionals, and the increasing emphasis on collecting data for evaluation and improvement, it is important to minimize respondent burden. This effort was catalyzed by a multifaceted initiative to redesign acute care delivery and promote seamless transitions of care, supported by the Center for Medicare $\&$ Medicaid Innovation. In initial testing, this questionnaire 
TABLE 1. Characteristics of the Respondents

\begin{tabular}{|c|c|c|c|}
\hline Characteristics & Total & $\mathrm{JHH}(\mathrm{N}=612)$ & JHBMC $(\mathrm{N}=229)$ \\
\hline \multicolumn{4}{|l|}{ Department } \\
\hline Medicine & $330(39.2 \%)$ & $234(38.2 \%)$ & 96 (41.9\%) \\
\hline Surgery & 248 (29.5\%) & $222(36.3 \%)$ & $26(11.4 \%)$ \\
\hline Neurology/Neurosciences & $90(10.7 \%)$ & $62(10.1 \%)$ & 28 (12.2\%) \\
\hline Psychiatry & $21(2.5 \%)$ & $14(2.3 \%)$ & $7(3.1 \%)$ \\
\hline Rehabilitation & $38(4.5 \%)$ & $30(4.9 \%)$ & $8(3.5 \%)$ \\
\hline Other & $60(7.1 \%)$ & $37(6.0 \%)$ & $20(8.7 \%)$ \\
\hline No response & $54(6.4 \%)$ & $13(2.1 \%)$ & $41(17.9 \%)$ \\
\hline \multicolumn{4}{|l|}{ Position } \\
\hline Nurse & $590(70.2 \%)$ & $416(68.0 \%)$ & $174(76.0 \%)$ \\
\hline Physician & $37(4.4 \%)$ & $23(3.8 \%)$ & $14(6.1 \%)$ \\
\hline Pharmacist & $16(1.9 \%)$ & $9(1.5 \%)$ & $7(3.1 \%)$ \\
\hline Dietitian/Nutritionist & $2(0.2 \%)$ & $2(0.3 \%)$ & 0 \\
\hline Physician Assistant & $10(1.2 \%)$ & $10(1.6 \%)$ & 0 \\
\hline Acute Care therapist & $35(4.2 \%)$ & $35(5.7 \%)$ & 0 \\
\hline Coordination staffa & $71(8.4 \%)$ & $53(8.7 \%)$ & $18(17.0 \%)$ \\
\hline Other & $22(2.6 \%)$ & $15(2.5 \%)$ & $7(3.1 \%)$ \\
\hline No response & $58(6.9 \%)$ & $49(8.0 \%)$ & $9(3.9 \%)$ \\
\hline \multicolumn{4}{|l|}{ Gender } \\
\hline Female & 702 (83.5\%) & $515(84.2 \%)$ & $187(81.7 \%)$ \\
\hline Male & $101(12.0 \%)$ & $75(12.3 \%)$ & $26(11.4 \%)$ \\
\hline No response & $38(4.5 \%)$ & $22(3.6 \%)$ & $16(6.9 \%)$ \\
\hline \multicolumn{4}{|l|}{ Total years in specialty } \\
\hline Less than 1 year & $76(9.0 \%)$ & $55(9.0 \%)$ & $21(9.2 \%)$ \\
\hline 1 to 5 years & 353 (42.0\%) & $275(44.9 \%)$ & 78 (34.1\%) \\
\hline 6 to 10 years & 138 (16.4\%) & $93(15.2 \%)$ & $45(20.0 \%)$ \\
\hline 11 years or more & 248 (29.5\%) & $173(28.3 \%)$ & 75 (32.8\%) \\
\hline No response & $26(3.1 \%)$ & $16(2.6 \%)$ & $10(4.4 \%)$ \\
\hline
\end{tabular}

${ }^{a}$ Coordination staff includes Case manager, Customer service representative, home care coordinator, social worker, transition guide, patient access line nurse and care coordination management staff. NOTE: Numbers are n (\%). Abbreviations: JHH; Johns Hopkins Hospital, JHBMC; Johns Hopkins Bayview Medical Center.

has evidence for reliability and validity. It was encouraging to find that the preliminary psychometric performance of the measure was very similar in 2 different settings of a tertiary academic hospital and a community hospital.

Our analysis of the survey data explored potential differences between the 2 hospitals, among different types of healthcare professionals and across different departments. Although we expected differences, we had no specific hypotheses about what those differences might be, and, in fact, did not observe any substantial differences. This could be taken to indicate that the intervention was uniformly and successfully implemented in both hospitals, and engaged various professionals in different departments. The ability to detect differences in care coordination at the nursing unit level could also prove to be beneficial for more precisely targeting where process improvement is needed. Further data collection and analyses should be conducted to more systematically compare units and to help identify those where practice is most advanced and those where improvements may be needed. It would also be informative to link differences in care coordination scores with patient outcomes. In addition, differences identified on specific domains between professional groups could be helpful to identify where great- er efforts are needed to improve interdisciplinary practice. Sampling strategies stratified by provider type would need to be targeted to make this kind of analysis informative.

The consistently lower scores observed for patient engagement, from the perspective of care professionals in all groups, suggest that this is an area where improvement is needed. These findings are consistent with published reports on the common failure by hospitals to include patients as a member of their own care team. In addition to measuring care processes from the perspective of frontline healthcare workers, future evaluations within the healthcare system would also benefit from including data collected from the perspective of the patient and family.

This study had some limitations. First, there may be more than 4 domains of care coordination that are important and can be measured in the acute care setting from provider perspective. However, the addition of more domains should be balanced against practicality and respondent burden. It may be possible to further clarify priority domains in hospital settings as opposed to the primary care setting. Future research should be directed to find these areas and to develop a more comprehensive, yet still concise measurement instrument. Second, the tool was developed to measure the impact of a 
TABLE 2. Item Completion Rate and Distribution, Factor Loadings, and Reliability

\begin{tabular}{|c|c|c|c|c|c|}
\hline Subscale & Item & Item Completion Rate & \% Ceiling & Factor Loading & Reliability \\
\hline \multirow{4}{*}{$\begin{array}{l}\text { Teamwork } \\
\text { (Eigenvalue: 5.28) }\end{array}$} & $\begin{array}{l}\text { 3. Our clinical leader alerts the healthcare team about } \\
\text { situations that may affect patient care. }\end{array}$ & $99.8 \%$ & $49.1 \%$ & 0.572 & \multirow{4}{*}{$\begin{array}{l}\text { Average inter-item correlation: } 0.476 \\
\text { Cronbach alpha: } 0.845\end{array}$} \\
\hline & $\begin{array}{l}\text { 4. Members of the healthcare team meet to reevaluate } \\
\text { the patient care plan when the patient's situation } \\
\text { had changed. }\end{array}$ & $99.7 \%$ & $35.0 \%$ & 0.619 & \\
\hline & $\begin{array}{l}\text { 5. The healthcare team uses input from multidisci- } \\
\text { plinary rounds to help determine the patient's care } \\
\text { plan. }\end{array}$ & $99.4 \%$ & $52.9 \%$ & 0.736 & \\
\hline & $\begin{array}{l}\text { 6. The healthcare team explains information to patients } \\
\text { and their families in lay terms. }\end{array}$ & $99.7 \%$ & $31.9 \%$ & 0.407 & \\
\hline Handoffs & $\begin{array}{l}\text { 7. My discipline has a clear protocol for sharing infor- } \\
\text { mation during patient handoffs. }\end{array}$ & $100 \%$ & $49.7 \%$ & $\mathrm{~N} / \mathrm{A}$ & $\mathrm{N} / \mathrm{A}$ \\
\hline \multirow{2}{*}{$\begin{array}{l}\text { Patient Engagement } \\
\text { (Eigenvalue: } 0.85 \text { ) }\end{array}$} & $\begin{array}{l}\text { 9. Patients are actively engaged in developing their } \\
\text { plan of care. }\end{array}$ & $98.6 \%$ & $18.3 \%$ & 0.864 & \multirow{2}{*}{$\begin{array}{l}\text { Average inter-item correlation: } 0.657 \\
\text { Cronbach alpha: } 0.852\end{array}$} \\
\hline & $\begin{array}{l}\text { 10. Patients are actively engaged in developing their } \\
\text { discharge plans. }\end{array}$ & $99.4 \%$ & $22.8 \%$ & 0.804 & \\
\hline \multirow[t]{2}{*}{$\begin{array}{l}\text { Transitions } \\
\text { (Eigenvalue: } 0.44 \text { ) }\end{array}$} & $\begin{array}{l}\text { 11. Members of the healthcare team teach patients } \\
\text { how to take care of themselves after they leave } \\
\text { the hospital. }\end{array}$ & $99.4 \%$ & $39.8 \%$ & 0.730 & \multirow[t]{2}{*}{$\begin{array}{l}\text { Average inter-item correlation: } 0.644 \\
\text { Cronbach alpha: } 0.856\end{array}$} \\
\hline & $\begin{array}{l}\text { 12. The healthcare team gives patients the tools they } \\
\text { need for a safe transition from the hospital to home, } \\
\text { or the next care setting. }\end{array}$ & $99.5 \%$ & $39.9 \%$ & 0.714 & \\
\hline
\end{tabular}

NOTE: \% Ceiling for subscales; $14.7 \%$ (Teamwork), 10.4\% (Patient Engagement), and 34.0\% (Transitions)

TABLE 3. Subscale Scores by Respondent Profession

\begin{tabular}{|c|c|c|c|c|c|}
\hline \multirow{2}{*}{ Scale } & \multirow{2}{*}{ Total $(\mathrm{N}=646)$} & \multicolumn{3}{|c|}{ Profession } & \multirow{2}{*}{$P$ value } \\
\hline & & Nurse $(\mathrm{N}=422)$ & Physician ( $N=36$ ) & Others $(N=188)$ & \\
\hline Teamwork & $4.19 \pm 0.71$ & $4.18 \pm 0.71$ & $4.25 \pm 0.69$ & $4.19 \pm 0.71$ & .856 \\
\hline Patient Engagement & $3.47 \pm 1.02$ & $3.40 \pm 1.05$ & $3.65 \pm 0.82$ & $3.60 \pm 0.95$ & .694 \\
\hline Transitions & $4.14 \pm 0.87$ & $4.19 \pm 0.85$ & $3.99 \pm 0.84$ & $4.06 \pm 0.89$ & .073 \\
\hline Handoffs & $4.22 \pm 0.99$ & $4.29 \pm 0.92$ & $4.03 \pm 1.27$ & $4.12 \pm 1.07$ & .316 \\
\hline Overall & $4.01 \pm 0.69$ & $4.00 \pm 0.70$ & $4.05 \pm 0.59$ & $4.01 \pm 0.68$ & .967 \\
\hline Global & $6.79 \pm 1.60$ & $6.71 \pm 1.61$ & $6.83 \pm 1.67$ & $6.96 \pm 1.57$ & .114 \\
\hline
\end{tabular}

NOTE: Numbers are mean \pm standard deviation. The scores for subscales range from 1 to 5 , and the scores for global scale range from 1 to 10 . Higher scores indicate better care coordination. $P$ values are generated from Kruskal-Wallis test.

large-scale intervention, and to fit into the specific context of 2 hospitals. Therefore, it should be tested in different settings of hospital care to see how it performs. However, virtually all hospitals in the United States today are adapting to changes in both financing and healthcare delivery. A tool such as the one described in this paper could be helpful to many organizations. Third, the scoring system for the overall scale score is not weighted and therefore reflects teamwork more than other components of care coordination, which are represented by fewer items. In general, we believe that use of the subscale scores may be more informative. Alternative scoring systems might also be proposed, including item weighting based on factor scores.

For the purposes of evaluation in this specific instance, we only collected data at a single point in time, after the intervention had been deployed. Thus, we were not able to evaluate the effectiveness of the J-CHiP intervention. We also did not intend to focus too much on the differences between units, giv- 


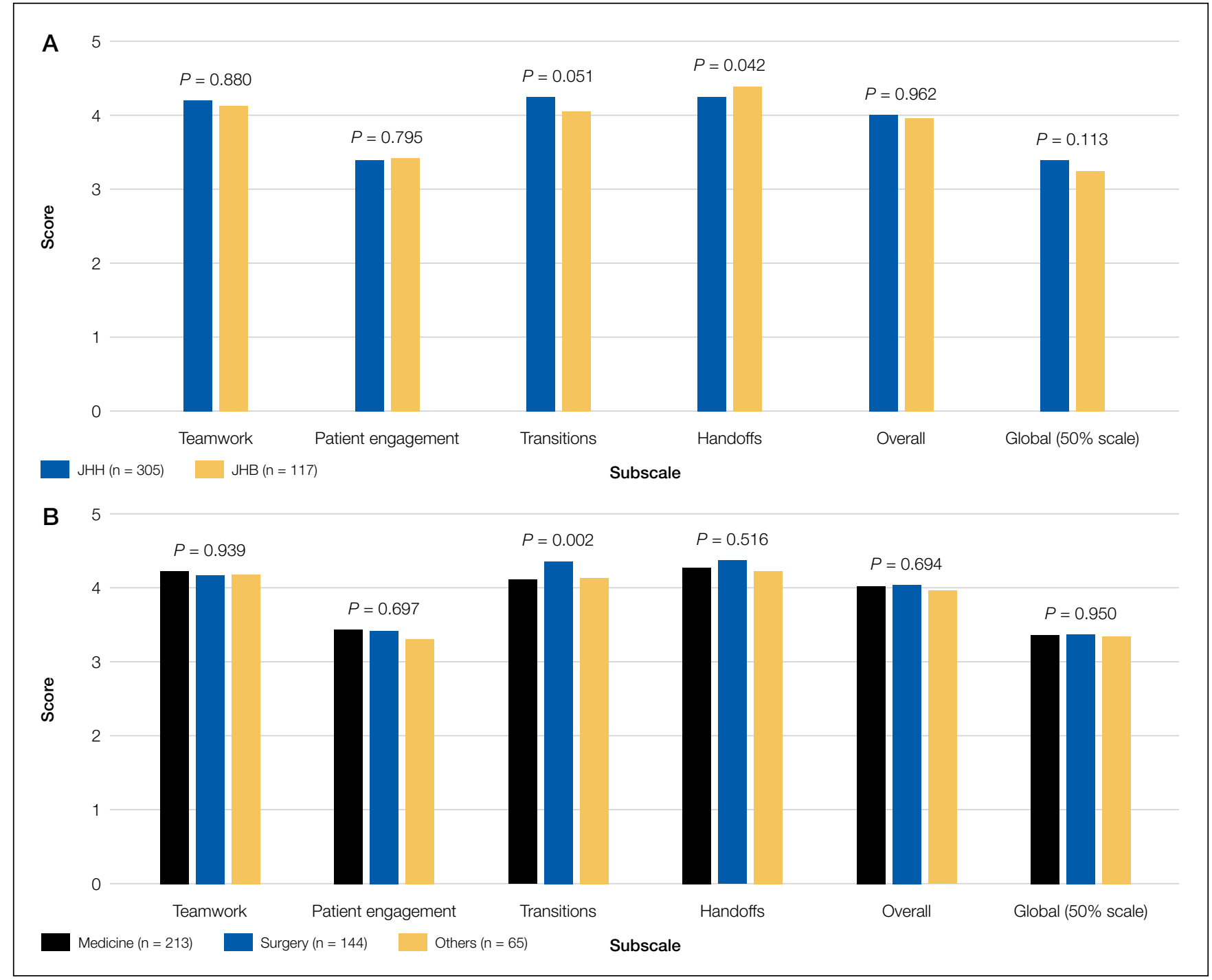

FIG. Mean subscale scores comparing nurses between hospitals (A) and departments (B). Subscale scores range from 1 to 5 . Global scores range from 1 to 10 and are shown here in $50 \%$ scale for visual comparison. Higher scores indicate better care coordination. $P$ values are generated from Wilcoxon rank sum test (A) and Kruskal-Wallis test (B).

en the limited number of respondents from individual units. It would be useful to collect more data at future time points, both to test the responsiveness of the scales and to evaluate the impact of future interventions at both the hospital and unit level.

The preliminary data from this study have generated insights about gaps in current practice, such as in engaging patients in the inpatient care process. It has also increased awareness by hospital leaders about the need to achieve high reliability in the adoption of new procedures and interdisciplinary practice. This tool might be used to find areas in need of improvement, to evaluate the effect of initiatives to improve care coordination, to monitor the change over time in the perception of care coordination among healthcare professionals, and to develop better intervention strategies for coordination activities in acute care settings. Additional research is needed to provide further evidence for the reliability and validity of this measure in diverse settings.
Disclosure: The project described was supported by Grant Number 1C1CMS331053-01-00 from the US Department of Health and Human Services, Centers for Medicare \& Medicaid Services. The contents of this publication are solely the responsibility of the authors and do not necessarily represent the official views of the US Department of Health and Human Services or any of its agencies. The research presented was conducted by the awardee. Results may or may not be consistent with or confirmed by the findings of the independent evaluation contractor.

The authors have no other disclosures.

\section{References}

1. McDonald KM, Sundaram V, Bravata DM, et al. Closing the Quality Gap: A Critical Analysis of Quality Improvement Strategies (Vol. 7: Care Coordination). Technical Reviews, No. 9.7. Rockville (MD): Agency for Healthcare Research and Quality (US); 2007.

2. Adams K, Corrigan J. Priority areas for national action: transforming health care quality. Washington, DC: National Academies Press; 2003.

3. Renders CM, Valk GD, Griffin S, Wagner EH, Eijk JT, Assendelft WJ. Interventions to improve the management of diabetes mellitus in primary care, outpatient and community settings. Cochrane Database Syst Rev. 2001(1):CD001481.

4. McAlister FA, Lawson FM, Teo KK, Armstrong PW. A systematic review of 
randomized trials of disease management programs in heart failure. Am J Med. 2001;110(5):378-384.

5. Bruce ML, Raue PJ, Reilly CF, et al. Clinical effectiveness of integrating depres sion care management into medicare home health: the Depression CAREPATH Randomized trial. JAMA Intern Med. 2015;175(1):55-64.

6. Berkowitz SA, Brown P, Brotman DJ, et al. Case Study: Johns Hopkins Community Health Partnership: A model for transformation. Healthc (Amst). 2016;4(4): $264-270$
7. McDonald. KM, Schultz. E, Albin. L, et al. Care Coordination Measures Atlas Version 4. Rockville, MD: Agency for Healthcare Research and Quality; 2014.

8 Schultz EM, Pineda N, Lonhart J, Davies SM, McDonald KM. A systematic review of the care coordination measurement landscape. BMC Health Serv Res. 2013;13:119.

9. Sexton JB, Helmreich RL, Neilands TB, et al. The Safety Attitudes Questionnaire: psychometric properties, benchmarking data, and emerging research. BMC Health Serv Res. 2006;6:44. 